\title{
Defination of length-scale parameter in Eringen's Nonlocal Elasticity via Nolocal Lattice and Finite Element Formulation
}

\author{
Büşra Uzun ${ }^{a}$, Hayri Metin Numanoğlu ${ }^{b}$, Ömer Civalek ${ }^{c^{*}}$ \\ ${ }^{a}$ Uluda ̆ University, Civil Engineering Department, Bursa, TURKIYE \\ ${ }_{b, c}$ Akdeniz University, Civil Engineering Department, Antalya, TURKIYE \\ "E-mail address: uzunbusra34@gmail.com ${ }^{a}$, metin_numanoglu@hotmail.comb ${ }^{b}$, civalek@yahoo.com ${ }^{\text {c* }}$
}

Received date: 10.10 .2018

Accepted date: 03.11.2018

ORCID numbers of authors:

0000-0002-7636-7170, $0000-0003-0556-7850^{b}, 0000-0003-1907-9479^{c}$

\begin{abstract}
Nonlocal elasticity theory is one of the popular approaches for nano mechanic problems. In this study, nonlocal parameter is defined via different approach. Nonlocal finite element formulations for axial vibration of nanorods have been given and some parameters are compared with the lattice dynamics. Weak form and final finite element formulation for axial vibration case have been derived.
\end{abstract}

Keywords: Nonlocal elasticity, finite element, axial vibration, rod, lattice dynamics.

\section{Introduction}

It is known that the general forms of the nonlocality are as follows [1]:

$$
\begin{gathered}
t_{k l, k}+\rho\left(f_{1}-\ddot{u}_{1}\right)=0 \\
t_{k l}(x)=\int_{V} \alpha\left(\left|x^{\prime}-x\right|, \tau\right) \sigma_{k l}\left(x^{\prime}\right) d v\left(x^{\prime}\right), \\
\sigma_{k l}\left(x^{\prime}\right)=\lambda e_{r r}\left(x^{\prime}\right) \delta_{k l}+2 \mu e_{k l}\left(x^{\prime}\right), \\
e_{k l}\left(x^{\prime}\right)=\frac{1}{2}\left(\frac{\partial u_{k}\left(x^{\prime}\right)}{\partial x_{l}^{\prime}}+\frac{\partial u_{l}\left(x^{\prime}\right)}{\partial x_{k}^{\prime}}\right),
\end{gathered}
$$


If the Eq. (2) is write in Eq. (1), we obtain

$$
\begin{gathered}
\frac{\partial \alpha}{\partial x_{k}} \sigma_{k l}\left(x^{\prime}\right)=-\frac{\partial \alpha}{\partial x_{k}^{\prime}} \sigma_{k l}\left(x^{\prime}\right) \\
=-\frac{\partial}{\partial x_{k}^{\prime}}\left[\alpha \sigma_{k l}\left(x^{\prime}\right)\right]+\alpha \frac{\partial \sigma_{k l}}{\partial x_{k}^{\prime}}-\int_{\partial V} \alpha\left(\left|x^{\prime}-x\right|\right) \sigma_{k l}\left(x^{\prime}\right) n_{k}^{\prime} d a\left(x^{\prime}\right)+\int_{V} \alpha\left(\left|x^{\prime}-x\right|\right) \\
\times \sigma_{k l, k} d v\left(x^{\prime}\right)+\rho\left(f_{1}-\ddot{u}_{1}\right)=0
\end{gathered}
$$

Here, the first integral through the surface represents the surface stresses. As a result, nonlocal elasticity theory includes surface physics, an important entity not included in classical theories. Again Eq. (3) and (4) introduce in Eq.(5), ones obtain

$$
\begin{aligned}
& -\int_{\partial V} \alpha\left(\left|x^{\prime}-x\right|\right)\left[\lambda u_{r, r}^{\prime} \delta_{k l}+\mu\left(u_{k, l}^{\prime}+u^{\prime} k\right)\right] n_{k}^{\prime} d a^{\prime}+\int_{V} \alpha\left(\left|x^{\prime}-x\right|\right) \\
& {\left[(\lambda+\mu) u_{k, l k}^{\prime}+\mu u_{l, k k}^{\prime}\right] d v^{\prime}+\rho\left(f_{1}-\ddot{u}_{1}\right)=0}
\end{aligned}
$$

In above equations the (') means depending on $x^{\prime}$. Namely, $u^{\prime}=u\left(x^{\prime}\right)$. If we solve the Eq.(6) under the suitable boundary and initial conditions, $u(x, t)$ displacement vector can be obtained. Initial conditions are depend $t_{k l}$ not $\sigma_{k l}$. So we can easily write $\tau_{\mathrm{kl}} n_{k}=t_{(n) l}$

\section{Definition of nonlocal parameter in nonlocal elasticity}

It is shown that the unit of nonlocal parameter located in Eq. (2) $\left(\alpha\left|x-x^{\prime}\right|\right)$ is (length) ${ }^{-3}$. Thus, the non-local parameter will be dependent on a characteristic length ratio $(a / l)$ in which an internal characteristic length a, (eg. lattice parameter, granular distance) and an external characteristic length $l$ (eg, crack length, wavelength) is present. Thus it defined as

$$
\alpha=\left(\alpha\left|x^{\prime}-x\right|, \tau\right), \tau=\frac{e_{0} a}{l}
$$

Here $e_{0}$ will be different constant for each material. Some properties of nonlocal parameter are as follows:

- It reach the maximum value at $x^{\prime}=x$ and decrease the value via $\left|x^{\prime}-x\right|$ calculation.

- When $\tau \rightarrow 0$, the statement of $\alpha$ is become Dirac delta function. Hence, the boundary (limit) of the classical elasticity introduce the nearly zero value boundary of the internal length scale (a). Namely:

$$
\lim _{\tau \rightarrow 0}=\left(\alpha\left|x^{\prime}-x\right|, \tau\right)=\delta\left(\alpha\left|x^{\prime}-x\right|\right)
$$

So, it is easily said that " $a$ " is a delta array. 
- $\quad$ For small internal length scale value (such as $\tau \rightarrow 1$ ) nonlocal elasticity theory behave as atomic lattice dynamics.

By matching the wave distribution curves with the distribution curves of the atomic lattice dynamics (or experiments), we can determine "a"value for a certain material. Various forms have been obtained as a result of research [2-16]. Some of them are as follows:

One-dimensional parameter

$$
\begin{gathered}
\alpha(|x|, \tau)=\frac{1}{l \tau}\left(1-\frac{|x|}{l \tau}\right),|x| \leq l \tau \\
\alpha(|x|, \tau)=0, \quad|x| \geq l \tau \\
\alpha(|x|, \tau)=\frac{1}{2 l \tau} e^{-\frac{|x|}{l \tau}} \\
\alpha(|x|, \tau)=\frac{1}{l \sqrt{\pi \tau}} e^{\frac{-x^{2}}{l^{2} \tau}}
\end{gathered}
$$

Two-dimensional parameter

$$
\alpha(|x|, \tau)=\frac{1}{2 \pi l^{2} \tau^{2}} K_{0}\left(\frac{\sqrt{x \cdot x}}{l \tau}\right)
$$

Here $K_{0}$ is a modified Bessel function.

Three-dimensional parameter

$$
\begin{aligned}
& \alpha(|x|, t)=\frac{1}{8(\pi t)^{3 / 2}} e^{-\frac{x \cdot x}{4 t}}, t=\frac{l^{2} \tau}{4} \\
& \alpha(|x|, \tau)=\frac{1}{4 \pi l^{2} \tau^{2} \sqrt{x \cdot x}} e^{-\frac{\sqrt{x \cdot x}}{\tau t}}
\end{aligned}
$$

When the equation 10 is examined, it is seen that the one-dimensional plane waves based on the Born-Kármán model, which is based on the theory of non-local elasticity and atomic lattice dynamics, fits perfectly with the distribution curve. When the two-dimensional parameter is analyzed, it is seen that the maximum error is $1.2 \%$ [1]. It is seen that all non-local parameters are normalized when the integrals are taken (over the length, area or volume). In addition, for 
$\tau \rightarrow 0$ the Dirac delta function is obtained. With this feature, it is seen that when the term Dirac delta function is used in Equation 2, classical theory of elasticity is reverted and Hooke's law becomes valid. This observation was developed by Eringen [2] as follows:

If $\alpha$ is the linear differential operator Green function, we write

$$
L \alpha\left(\left|x^{\prime}-x\right|, \tau\right)=\delta\left(\left|x^{\prime}-x\right|\right)
$$

After used this Equation in Eq.(2), ones obtain

$$
L t_{k l}=\sigma_{k l}
$$

Let be consider the $\mathrm{L}$ is a differential operator having constant coefficients

$$
\left(L t_{k l}\right)_{, k}=L t_{k l, k}
$$

So, we obtain the below equation

$$
\sigma_{k l, k}+L \rho\left(f_{1}-\ddot{u}_{1}\right)=0
$$

Hence we obtain the differential equation instead of partial integral. For static case $L \rho\left(f_{1}-\ddot{u}_{1}\right)=0$

Finally, we can write below form

$$
\sigma_{k l, k}=0
$$

If we sued the Eq. (19) in Eq.(3) we obtain the well-known Navier equation. So, differential operator as define via Eq.(3)

$$
L=1-\tau^{2} l^{2} \nabla^{2}
$$

After using this equation in Eq. (17) we obtained the following form

$$
\left(1-\tau^{2} l^{2} \nabla^{2}\right) \cdot t=\sigma
$$

The accuracy of this result can be demonstrated by the atomic distribution relationship. For this purpose, the frequency expression obtained from the Born-Kármán model must be equal to the expression of non-local elasticity for plane waves.

\section{Modeling by lattice dynamics}

Lattice dynamics is known as harmonic approach provided that the displacements are small. In the chain, atoms can be connected with elastic springs (Figure 1). 

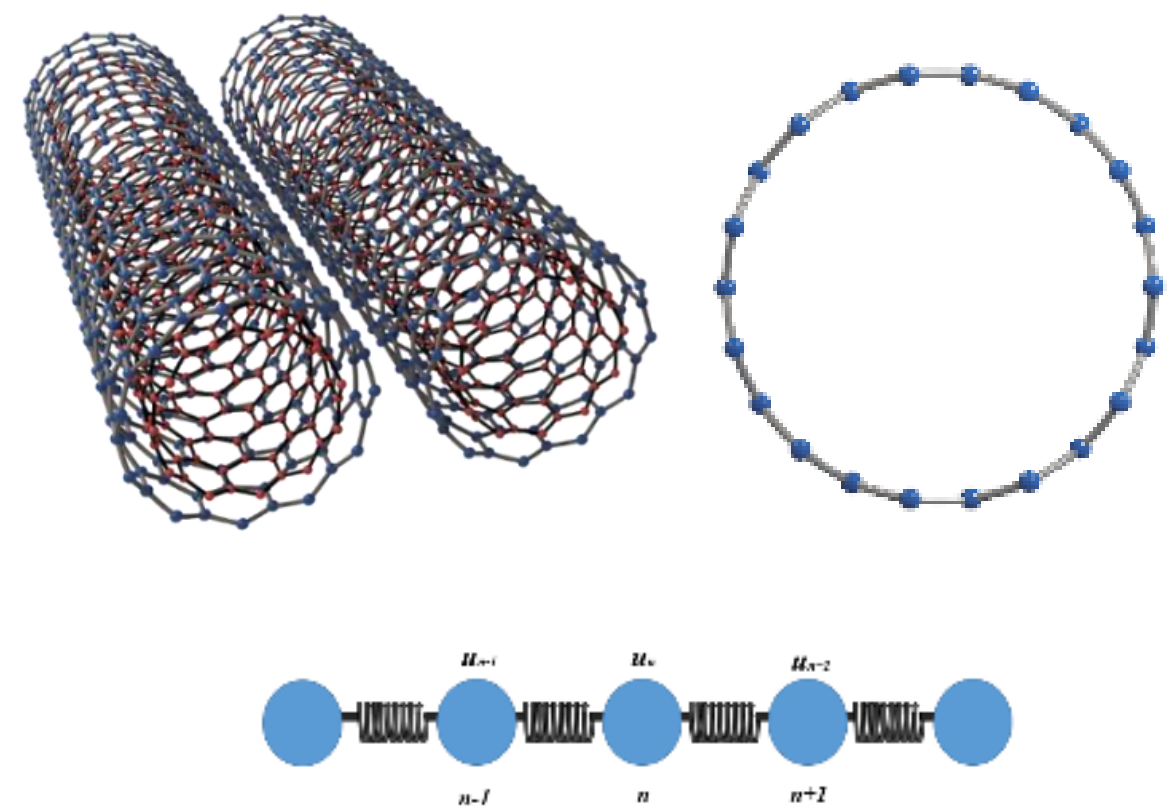

Fig. 1. One-dimensional lattice model

Therefore, force applied to the $n^{\text {th }}$ atom can be written as

$$
F_{n}=K\left(u_{n+1}-u_{n}\right)+K\left(u_{n-1}-u_{n}\right)
$$

Here $\mathrm{K}$ is the inter-atomic force (elastic) constant. Newton's second law applied to the $n^{\text {th }}$ atom

$$
M \frac{d^{2} u_{n}}{d t^{2}}=F_{n}=K\left(u_{n+1}-u_{n}\right)+K\left(u_{n-1}-u_{n}\right)=-K\left(2 u_{n}-u_{n+1}-u_{n-1}\right)
$$

In the above expression $\mathrm{M}$ denotes the mass of the atom. Similarly, the equation for each atom in the cage should be written. $\mathrm{N}$; In order to express the total number of atoms, the result is the $\mathrm{N}$ equation which must be solved simultaneously. In addition, the boundary conditions applied to the end of the cage must also be taken into account. The following conversion will be used for the solution

$$
u_{n}=A e^{i\left(k x_{n}-\omega t\right)}
$$

Here, $x_{n} \mathrm{n}$. refers to the position of the atom and $x_{n}=n a$. This equation represents a moving wave with $q$ wavelength where all atoms oscillate at the same frequency $(q)$ with the same $A$ amplitude. Equation (24) is written in Equation (23)

$$
M\left(-\omega^{2}\right) e^{i k n a}=-C\left(2 e^{i k n a}-e^{i k(n+1) a}-e^{i k(n-1) a}\right)
$$

After some manipulation 


$$
\begin{gathered}
M \omega^{2}=C\left(2-e^{i k a}-e^{-i k a}\right)=2 C(1-\cos k a) \\
\frac{M}{C} \omega^{2}=2(1-\cos k a)
\end{gathered}
$$

Finally

$$
\frac{M}{C} \omega^{2}=2(1-\cos k a)=4 \sin ^{2} \frac{k a}{2}
$$

So, frequency and distribution relation is

$$
\sqrt{\omega_{j}^{2}}=\sqrt{\frac{M}{C} \omega^{2}}=2\left|\sin \frac{k a}{2}\right|
$$

We expect the results obtained with the cage dynamics between the two atoms to be the same as the non-local elasticity results. In this case, the ratio of cage dynamics frequency distribution relation to bar frequency distribution relation

$$
\alpha(k)=\frac{\omega_{j}^{2}(k)}{\omega_{o j}{ }^{2}}=\left(\frac{2 \kappa}{\pi k}\right)^{2} \sin ^{2}\left(\frac{\pi k}{2 \kappa}\right)
$$

Here angular frequency can be write as

$$
\omega_{o j}^{2}=c_{0}^{2} k^{2}
$$

Also $c_{0}=\sqrt{E / \rho} \cdot \kappa$ is the Brillouin region is the value of the upper limit of $\mathrm{k}$. This value is for one dimensional mesh dynamics. In the light of this information Equation (31) is reorganized

$$
\alpha(k)=\frac{\omega_{j}{ }^{2}(k)}{\omega_{o j}{ }^{2}}=\left(\frac{2}{k a}\right)^{2} \sin ^{2}\left(\frac{k a}{2}\right)
$$

If we write above equation as terms of cosine

$$
\alpha(k)=\frac{{\omega_{j}^{2}}^{2}(k)}{{\omega_{o j}^{2}}^{2}}=\frac{2}{k^{2} a^{2}}(1-\cos k a)
$$

If the equation opens into the Maclaurin series 


$$
\begin{aligned}
& \cos k a=1-\frac{(k a)^{2}}{2 !}+\frac{(k a)^{4}}{4 !}-\frac{(k a)^{6}}{6 !}+\ldots \\
& 1-\cos k a=\frac{(k a)^{2}}{2 !}-\frac{(k a)^{4}}{4 !}+\frac{(k a)^{6}}{6 !}-\ldots
\end{aligned}
$$

After using these equations in Eq.(32), we obtain

$$
\alpha(k)=\frac{2}{(k a)^{2}}(1-\cos k a)=1-\frac{(k a)^{2}}{12}+\frac{(k a)^{4}}{360}-\ldots
$$

Using the first-order approach and by using the first two-term of the Eq.36

$$
\frac{1}{\alpha}=1+\left(e_{0} a\right)^{2} k^{2}
$$

After some arrangement

$$
\alpha=\left(1+e_{0}^{2} a^{2} k^{2}\right)^{-1},\left(1+e_{0}^{2} a^{2} k^{2}\right) \bar{t}_{k l}=\bar{\sigma}_{k l},\left(1-e_{0}^{2} a^{2} \nabla^{2}-\ldots\right) t_{k l}=\sigma_{k l}
$$

Hence, non-dimensional frequency via nonlocal elasticity is

$$
\frac{\omega a}{c_{0}}=k a\left(1+e_{0}^{2} a^{2} k^{2}\right)^{-1 / 2}
$$

The relation of frequency distribution via lattice dynamics is as follows with the help of equation (29)

$$
\frac{\omega a}{c_{0}}=2 \sin (k a / 2)
$$

If the Eq. (39) and Eq. (40) are equalized for $k a=\pi$

$$
\begin{gathered}
k a\left(1+e_{0}^{2} a^{2} k^{2}\right)^{-1 / 2}=2 \sin (k a / 2) \\
\pi\left(1+e_{0}^{2} \pi^{2}\right)^{-1 / 2}=2 \sin (\pi / 2) \\
e_{0}=0.39
\end{gathered}
$$

If second-order approach Eq. (36) extract the polynomial form in three-terms as 


$$
\frac{1}{\alpha}=1+\left(e_{0} a\right)^{2} k^{2}+\left(\gamma_{0} a\right)^{4} k^{4}, \alpha=\left(1+e_{0}^{2} a^{2} k^{2}+\gamma_{0}{ }^{4} a^{4} k^{4}\right)^{-1}
$$

And nonlocal stress equation

$$
\left(1+e_{0}^{2} a^{2} k^{2}+\gamma_{0}^{4} a^{4} k^{4}\right) \bar{t}_{k l}=\bar{\sigma}_{k l}
$$

In this last equation $\bar{t}_{k l}$ and $\bar{\sigma}_{k l}$, means the Fourier transforms of $t_{k l}$ and $\sigma_{k l}$, , respectively. If we take the inverse Fourier transform of Eq. (43)

$$
\left(1-e_{0}^{2} a^{2} \nabla^{2}+\gamma_{0}^{4} a^{4} \nabla^{4}-\ldots\right) t_{k l}=\sigma_{k l}
$$

Also, the governing equation for stress in nonlocal case is

$$
\sigma_{k l, k}+\left(1-e_{0}^{2} a^{2} \nabla^{2}+\gamma_{0}^{4} a^{4} \nabla^{4}\right)\left(\rho f_{l}-\rho \ddot{u}_{l}\right)=0
$$

These equations replaced Navier's classical elasticity equations. New dimensionless frequency according to non-local elasticity theory is

$$
\frac{\omega a}{c_{0}}=k a\left(1+e_{0}^{2} a^{2} k^{2}+\gamma_{0}^{4} a^{4} k^{4}\right)^{-1 / 2}
$$

Lazar et al. [16]. Stated that $\varepsilon^{4}=4 \gamma^{4}$. Frequency distribution relationship via lattice dynamics is as follows

$$
\frac{\omega a}{c_{0}}=2 \sin (k a / 2)
$$

Eq. (46) and Eq. (47) are equalized for $k a=\pi$

$$
\begin{gathered}
k d\left(1+e_{0}^{2} a^{2} k^{2}+\gamma_{0}{ }^{4} a^{4} k^{4}\right)^{-1 / 2}=2 \sin (k a / 2) \\
\pi\left(1+2 \gamma_{0}^{2} \pi^{2}+\gamma_{0}{ }^{4} \pi^{4}\right)^{-1 / 2}=2 \sin (\pi / 2) \\
\gamma_{0}=0.24, \varepsilon_{0}=0.339
\end{gathered}
$$




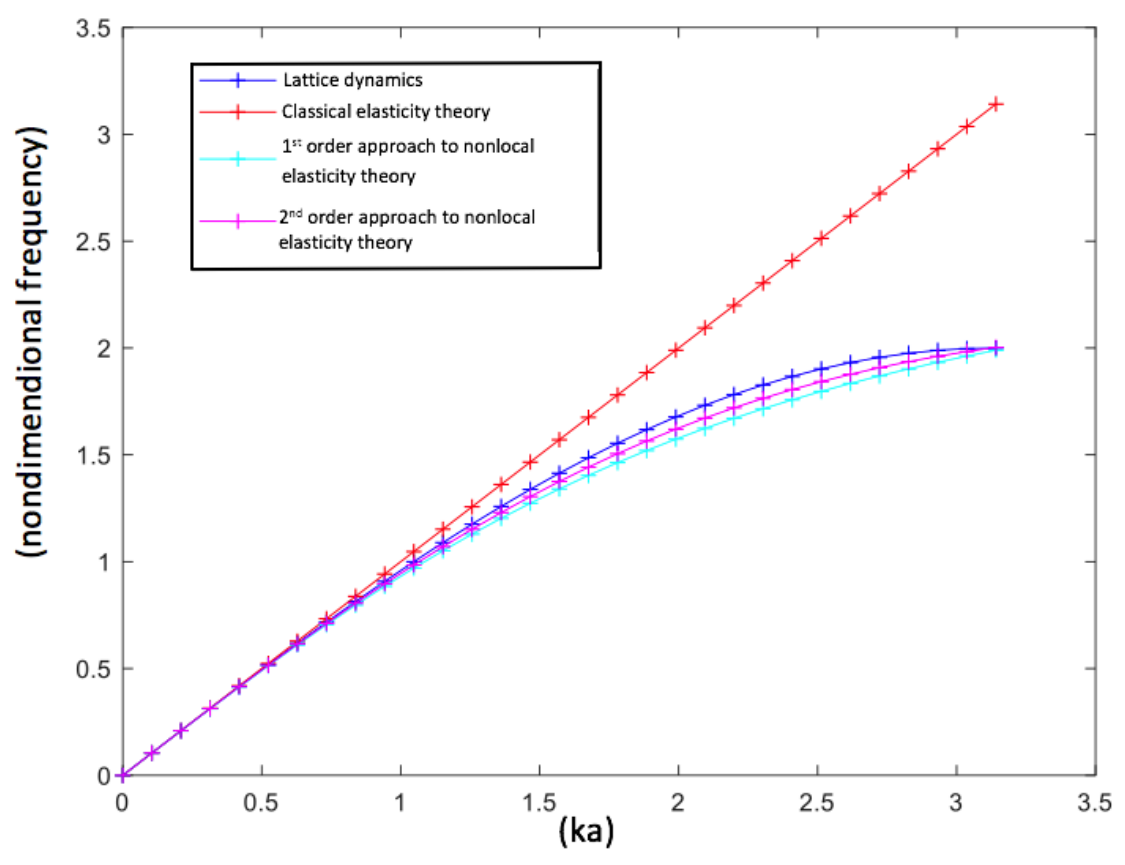

Fig. 2. Convergence of frequency value

The first and secon-order approaches of the theory of non-local elasticity, classical elasticity theory, and the frequency comparison of the lattice dynamics are presented in Figure 2. As can be seen from the figure, the frequency results for the second order approximation of nonlocal elasticity theory are closer to the lattice dynamic than the first-order approach. In more general case (under the axial foundation effect and thermal effect) free vibration form of axial vibration of elastic nanorod have been given as two different forms:

$$
\begin{aligned}
& E A \frac{\partial^{2} u}{\partial x^{2}}=(E A \alpha \Delta T)^{\prime}-f(x)+k_{w} u(x)+\rho A \frac{\partial^{2} u}{\partial t^{2}} \\
& +\left(e_{0} a\right)^{2} \frac{\partial^{2}}{\partial x^{2}}\left(f-k-\rho A \frac{\partial^{2} u}{\partial t^{2}}\right) \\
& \int_{0}^{L}\left[\begin{array}{l}
\left.E A \frac{\partial u}{\partial x} \frac{\partial w}{\partial x}+E A \alpha_{T} \Delta_{T} \frac{\partial w}{\partial x}+w f(x)-w k u(x)-\rho A w \frac{\partial^{2} u}{\partial t^{2}}\right] d x \\
-\mu f(x) \frac{\partial^{2} w}{\partial x^{2}}-\mu k \frac{\partial w}{\partial x} \frac{\partial u}{\partial x}-\mu \rho A \frac{\partial^{3} u}{\partial x \partial t^{2}} \frac{\partial w}{\partial x}
\end{array}\right]
\end{aligned}
$$

Eq. (50) is the weak form for FEM approach of axial vibration. Some applications have also been listed in references related to macro, micro and nanomechanics [17-39]. 


\section{Conclusion}

Some comparison has been made for axial vibration. First and second order approach for nonlocal elasticity and lattice dynamics results have also been compared. Finally, weak form is given for axial vibration problem of nanorods.

\section{Acknowledgements}

This study has been supported by The Scientific and Technological Research Council of Turkey (TÜBITAK) with Project no: 117M495. This support is gratefully acknowledged.

\section{References}

[1] Ari, N., Eringen A.C., Nonlocal Stress-Field at Griffith Crack, Crystal Lattice Defects and Amorphous Materials, 10(1), 33-38, 1983.

[2] Eringen, A. C., Linear theory of micropolar elasticity, Journal of Mathematics and Mechanics, 909-923, 1966.

[3] Eringen, A. C., A unified theory of thermomechanical materials, International Journal of Engineering Science, 4(2), 179-202, 1966.

[4] Eringen, A. C., Micropolar fluids with stretch, International Journal of Engineering Science, 7(1), 115-127, 1969.

[5] Eringen, A. C., Linear Theory of Nonlocal Elasticity and Dispersion of Plane-Waves, International Journal of Engineering Science, 10(5), 425-435, 1972.

[6] Thoft-Christensen, P., Continuum Mechanics Aspects of Geodynamics and Rock Fracture Mechanics: Proceedings of the NATO Advanced Study Institute held in Reykjavik, Iceland, 11-20 August, 1974. (Vol. 12), Springer Science \& Business Media, 2012.

[7] Eringen, A. C., Nonlocal Polar Elastic Continua, International Journal of Engineering Science, 10(1), 1-16, 1972. doi: 10.1016/0020-7225(72)90070-5

[8] Eringen, A. C., On nonlocal fluid mechanics, International Journal of Engineering Science, 10(6), 561-575, 1972.

[9] Eringen, A. C., Nonlocal polar field theories, Continuum physics, 4(Part III), 205-264, 1976.

[10] Eringen, A. C., Screw Dislocation in Nonlocal Elasticity, Journal of Physics D-Applied Physics, 10(5), 671-678, 1976. doi: 10.1088/0022-3727/10/5/009.

[11] Eringen, A. C., On differential equations of nonlocal elasticity and solutions of screw dislocation and surface waves, Journal of applied physics, 54(9), 4703-4710, 1983.

[12] Eringen, A. C., Nonlocal continuum field theories, Springer Science \& Business Media, 2002.

[13] Eringen, A. C., Edelen, D. G. B., On nonlocal elasticity, International Journal of Engineering Science, 10(3), 233-248, 1972.

[14] Eringen, A. C., Kim, B. S., Stress concentration at the tip of crack, Mechanics Research Communications, 1(4), 233-237, 1974. 
[15] Eringen, A. C., Kim, B. S., Relation between Nonlocal Elasticity and Lattice-Dynamics, Crystal Lattice Defects, 7(2), 51-57, 1977.

[16] Lazar, M., Maugin, G. A., Aifantis, E. C., On a theory of nonlocal elasticity of biHelmholtz type and some applications, International Journal of Solids and Structures, 43(6), 1404-1421, 2006.

[17] Mercan, K., Civalek, O., DSC method for buckling analysis of boron nitride nanotube (BNNT) surrounded by an elastic matrix, Composite Structures, 143, 300-309, 2016.

[18] Baltacioglu, A. K., Akgoz, B., Civalek, O., Nonlinear static response of laminated composite plates by discrete singular convolution method, Composite Structures, 93, 153 $161,2010$.

[19] Civalek, O, Application of differential quadrature (DQ) and harmonic differential quadrature (HDQ) for buckling analysis of thin isotropic plates and elastic columns, Engineering Structures, 26, 171-186, 2004.

[20] Demir, C., Mercan, K., Civalek, O., Determination of critical buckling loads of isotropic, FGM and laminated truncated conical panel, Composites Part B, 94, 1-10, 2016.

[21] Civalek, O., Finite Element analysis of plates and shells, Elazı̆̆, Firat University, 1998.

[22] Phadikar, J. K., Pradhan, S. C., Variational formulation and finite element analysis for nonlocal elastic nanobeams and nanoplates, Computational materials science, 49(3), 492499, 2010.

[23] Mercan, K., Ersoy, H., Civalek, Ö., Free vibration of annular plates by discrete singular convolution and differential quadrature methods, Journal of Applied and Computational Mechanics, 2(3), 128-133, 2016.

[24] Akgöz, B., Civalek, O., Buckling analysis of cantilever carbon nanotubes using the strain gradient elasticity and modified couple stress theories, Journal of Computational and Theoretical Nanoscience, 8(9), 1821-1827, 2011.

[25] Chen, Y., Lee, J. D., Eskandarian, A., Atomistic viewpoint of the applicability of microcontinuum theories, International Journal of Solids and Structures, 41(8), 20852097, 2004.

[26] Baltacioglu, A.K., Civalek, O., Akgoz, B., Demir, F., Large deflection analysis of laminated composite plates resting on nonlinear elastic foundations by the method of discrete singular convolution, International Journal of Pressure Vessels and Piping, 88, 290-300, 2011.

[27] Omurtag, M. H., Çubuk sonlu elemanlar, Birsen Yayınevi, 2010.

[28] Civalek, O., Free vibration of carbon nanotubes reinforced (CNTR) and functionally graded shells and plates based on FSDT via discrete singular convolution method, Composites Part B, 111, 45-59, 2017.

[29] Peddieson, J., Buchanan, G. R., McNitt, R. P., Application of nonlocal continuum models to nanotechnology, International Journal of Engineering Science, 41(3), 305-312, 2003.

[30] Civalek, O., Nonlinear dynamic response of laminated plates resting on nonlinear elastic foundations by the discrete singular convolution-differential quadrature coupled approaches. Composites: Part B, 50, 171-179, 2013. 
[31] Mercan, K., Civalek, O., Buckling analysis of Silicon carbide nanotubes (SiCNTs) with surface effect and nonlocal elasticity using the method of HDQ, Composites Part B, 114, 34-45, 2017.

[32] Chen, W. J., Li, X. P., Size-dependent free vibration analysis of composite laminated Timoshenko beam based on new modified couple stress theory, Archive of Applied Mechanics, 83, 431-444, 2013.

[33] Civalek, O., Demir, C., Buckling and bending analyses of cantilever carbon nanotubes using the euler-bernoulli beam theory based on non-local continuum model, Asian Journal of Civil Engineering, 12(5), 651-661, 2011.

[34] Narendar, S., Gopalakrishnan, S., Nonlocal scale effects on ultrasonic wave characteristics of nanorods, Physica E: Low-dimensional Systems and Nanostructures, 42(5), 1601-1604, 2010.

[35] Civalek, O., Geometrically non-linear static and dynamic analysis of plates and shells resting on elastic foundation by the method of polynomial differential quadrature (PDQ), Elazığg, Firat University, 2004.

[36] Houari, M. S. A., Bessaim, A., Bernard, F., Tounsi, A., Mahmoud, S. R., Buckling analysis of new quasi-3D FG nanobeams based on nonlocal strain gradient elasticity theory and variable length scale parameter, Steel and Composite Structures, 28, 13-24, 2018.

[37] Narendar, S., Nonlocal thermodynamic response of a rod, Journal of Thermal Stresses, 40(12), 1595-1605, 2017.

[38] Karlicic, D., Murmu, T., Adhikari, S., Mccarthy, M., Non-local structural mechanics, John Wiley \& Sons, 2015.

[39] Zhang, Y. Q., Liu, X., Liu, G. R., Thermal effect on transverse vibrations of double-walled carbon nanotubes, Nanotechnology, 18(44), 445701, 2007. 